\title{
Review Article \\ The Current Role of Lymph Node Dissection in the Management of Renal Cell Carcinoma
}

\author{
Joseph Edmund Jamal and Thomas William Jarrett \\ Department of Urology, The George Washington University Medical Faculty Associates, 2150 Pennsylvania Avenue, NW, \\ Washington, DC 20037, USA \\ Correspondence should be addressed to Joseph Edmund Jamal, jjamal@mfa.gwu.edu
}

Received 1 February 2011; Accepted 3 April 2011

Academic Editor: Timothy M. Pawlik

Copyright ( 92011 J. E. Jamal and T. W. Jarrett. This is an open access article distributed under the Creative Commons Attribution License, which permits unrestricted use, distribution, and reproduction in any medium, provided the original work is properly cited.

\begin{abstract}
The role of lymph node dissection remains controversial in the surgical management of renal cell carcinoma. Incidental renal masses are being diagnosed at increasing rates due to the routine use of CT scans. Despite the increase in incidental diagnosis of renal masses, $20 \%$ to $30 \%$ of patients present with metastatic disease. Currently, surgeons do not routinely perform lymph node dissection unless there is gross evidence of lymphadenopathy, as patients without clinical evidence of lymphadenopathy rarely have positive nodes at the time of surgery. Patients with metastatic disease to the regional lymph nodes have a poor overall prognosis. However, some evidence supports a therapeutic benefit of lymphadenectomy in these patients. Further, the staging information gained from diagnosing lymph node involvement may allow for the use of new agents to treat metastatic disease and effect outcomes.
\end{abstract}

\section{Introduction}

The role of lymph node dissection (LND) remains controversial in the surgical management of renal cell carcinoma (RCC) [1-3]. To date, there is no definitive data which indicates an overall survival advantage imparted by performing LND in patients with RCC. Additionally, a complete LND during radical nephrectomy (RN) adds significant time, potential morbidity, and requires dissection of and around the great vessels. What is clear is that in patients with renal cell carcinoma without evidence of distant metastases, the presence of lymph node-only metastases is associated with a poor prognosis [4-10]. For this reason, identifying patients at risk for positive lymph nodes at the time of surgical treatment of their renal mass remains essential.

Recently, the first prospective, randomized trial was conducted by the European Organization for Research and Treatment of Cancer (EORTC) to compare the long-term results of radical nephrectomy alone $(n=389)$ versus radical nephrectomy with complete lymphadenectomy $(n=$ 383) for patients with clinically N0, M0 disease [1]. They concluded that LND is not therapeutic in the routine management of RCC, but nor did it increase the morbidity of surgical management. This has spurred significant debate in the urologic oncology community $[3,11,12]$. Indeed, this study was aimed at determining the long-term outcomes in patients with clinically localized disease, and found that $70 \%$ of patients had pT1 or pT2 disease. The majority of these patients were therefore unlikely to benefit from LND in the first place. Therefore, it is important to emphasize that although being a landmark study, it does not negate the role for LND in RCC. With the recent availability of new adjuvant agents, prospective adjuvant protocol studies nearing completion, and neoadjuvant protocols currently under study, the importance of LND may change, either as a therapeutic or staging procedure. The topic of LND therefore requires further discourse.

In clinical practice, the role of LND in the management of RCC remains controversial due to variability of lymphatic drainage of the kidney, the absence of lymphatic involvement in many patients with disseminated disease, and the lack of definitive evidence of any survival benefit imparted by LND [1]. We address the current controversies regarding the role of lymphadenectomy in the surgical management 
of renal tumors in this review. Ultimately, the therapeutic benefit of LND in RCC, whether it be beneficial by itself or in that it may be diagnostic of metastatic disease resulting in initiation of adjuvant chemotherapy, may only be realized in a select subset of patients.

\section{Epidemiology and Relevance}

Renal cell carcinoma comprises $2 \%$ to $3 \%$ of malignant neoplasms in adults. In the United States, 31,000 new cases of RCC are diagnosed annually, and 11,900 patients die of disease $[13,14]$. There is a male-to-female ratio of $3: 2$ [13], and a $10 \%$ to $20 \%$ higher incidence in African Americans [15]. The majority of cases of RCC are believed to be sporadic, with only $4 \%$ familial according to National Cancer Institute estimates. Approximately $20 \%$ to $30 \%$ of patients with RCC present with metastatic disease [16], but ranges from $3 \%$ in surgical series to $63.6 \%$ in autopsy series [16]. Of these patients with metastatic disease, historically, $40 \%$ have distant metastases only without evidence of lymph node involvement, $50 \%$ have both distant metastases and lymph node involvement, and approximately 3-10\% present with lymph node involvement only [1, 17-19]. Additionally, one-third of patients with localized RCC will eventually develop recurrence or progression. There has been downward stage migration, as well as increasing incidence of RCC due to extensive utilization of cross-sectional imaging.

In combination, the clinical stage and pathologic grade of the tumor is highly predictive of positive lymph node metastases. When LND is performed, a number of studies have shown that positive lymph nodes have an independent adverse effect on outcome, irrespective of other variables [20-22]. Patients with node-positive disease have 5-year survival rates ranging from $5 \%$ to $35 \%$ [23].

\section{Templates for Lymph Node Dissection}

Renal lymphatic drainage is variable, adding to the controversy of performing LND at the time of RN. There is currently no consensus on the anatomic extent of LND. This makes it exceedingly difficult to compare studies as there is a clear lack of standardization. Further, many studies do not delineate the template used for LND. A "standard" LND for RCC on the left side includes the paraaortic and preaortic nodes from the crus of the diaphragm to the inferior mesenteric artery (IMA). On the right, a "standard" LND includes the paracaval and precaval nodes from the adrenal vein along the vena cava, also down to the level of the IMA. An "extended" LND adds the interaortocaval nodes down to the bifurcation of the great vessels on both sides, with the inclusion of retrocaval nodes for right-sided primary tumors.

Crispen and colleagues recently examined their series of patients with LND and evaluated the lymphatic drainage patterns for recommendations for surgical templates [24]. Of 169 patients who fit the criteria for LND, 64 (38\%) were found to have metastatic disease to their retroperitoneal lymph nodes, with the median of 6 lymph nodes removed with LND. For right-sided tumors, the primary lymphatic drainage was the paracaval, precaval, retrocaval, and interaortocaval lymph nodes. For left-sided tumors, the primary lymphatic drainage was the paraaortic, preaortic, retroaortic, and interaortocaval lymph nodes. They advocate for a standard removal of all the lymphatic tissue in these primary lymphatic landing zones. When examining their data of patients with positive nodal disease, left-sided tumors corresponded with $76 \%$ of left hilar and $62 \%$ of paraaortic positive lymph nodes (of patients with positive nodal disease). On the right side, patients with positive lymph node involvement were found to occur in $57 \%$ of paracaval and $43 \%$ of right hilar nodes. On both sides, lymph nodes from the renal hilum were not always involved in patients with nodal disease, supporting the argument against merely sampling the renal hilar lymph nodes as being sufficient. Based on these findings, they recommend that when lymphadenectomy is performed in patients without palpable disease, that left-sided disease LND includes paraaortic and interaortocaval lymph nodes, and right-sided disease LND includes paracaval and interaortocaval lymph nodes.

Morbidity of LND has largely been found to be minimal when compared to nephrectomy alone. Retrospective review has shown little difference in morbidity of LND [25]. Additionally, the recent prospective EORTC 30881 trial also showed no appreciable difference in morbidity between their two randomized groups. They did not comment on the additional length of time the LND added, nor the total number of lymph nodes removed. They included morbidities such as bleeding $>1$ liter, pleural injury, infection, bowel injury, embolism, and lymph fluid drainage, and found no significant differences between patients who had LND and those who did not. Siminovitch and colleagues [26] performed a direct comparison of extended, hilar, and regional LND templates. They found no differences in morbidity or survival rates between the three different LND templates. Regardless, LND remains a complex procedure, and carries risk for serious intraoperative complications. Great care must be taken when performing the procedure to ensure minimal morbidity.

\section{Indications and Assessment}

Indications for LND at the time of RN are not generally clear. Signs of lymphadenopathy or evidence of locally advanced disease on cross-sectional imaging, warrants LND. Palpable lymphadenopathy, or evidence of bulky lymph nodes with laparoscopy, at the time of $\mathrm{RN}$ can be indications for LND. Unfortunately, radiographic lymphadenopathy only modestly correlates with metastatic involvement, with $32 \%-$ $43 \%$ of nodes $>1 \mathrm{~cm}$ harboring cancer $[27,28]$. Other studies have shown that $16 \%-42 \%$ of lymph nodes suspicious on are falsely positive $[1,18,29]$. Many of these nodes are inflammatory in nature, and therefore no benefit is imparted by their removal. The difficulties lie in determining the lymphatic drainage patterns of each kidney, which lymph nodes are potentially positive, and to what extent a lymphadenectomy should be performed. 
Nomograms have been developed to aid in risk stratification. One nomogram had 78\% accuracy by incorporating patient age, radiographic tumor size, and symptoms [28]. Another preoperative nomogram, which included 4844 patients, found a concordance index of 0.76 when including symptoms at presentation, radiographic lymphadenopathy, tumor size, and hematuria [30]. Neither of these nomograms have gained widespread acceptance.

Crispen and colleagues evaluated the performance of LND in high-risk clear cell RCC patients between 2002 and 2006 [24]. They had previously identified five intraoperative pathological features which were considered high-risk for nodal metastases, and performed LND if at least two of these risk factors were present intraoperatively. These were nuclear grade 3 or 4 , sarcomatoid component, tumor size $\geq 10 \mathrm{~cm}$, tumor stage pT3 or pT4, and coagulative tumor necrosis. Of 169 RNs that had 2 high-risk intraoperative factors, 63 (38\%) had nodal metastases.

Others have novel approaches to expanding indications for LND. Bex and colleagues evaluated the role of sentinel node detection in patients with RCC [31]. Previous series indicate that $58-95 \%$ of patients with lymph node disease have associated hematogenous spread $[32,33]$, prompting Bex and colleagues to assess the feasibility of identifying a sentinel node to aid in staging. Eight patients with $\mathrm{cT} 1 \mathrm{cN} 0$ cM0 RCC had their tumors injected (percutaneously under ultrasound guidance) with a radionuclide tracer, ${ }^{99 \mathrm{~m}} \mathrm{Tc}$ nanocolloid. Both lymphoscintigraphy and SPECT/CT were performed to determine the anatomic location of the sentinel node, as there is variable lymphatic drainage of the kidney. Surgery was then performed the following day utilizing intraoperative gamma probes to identify the radioactive nodes. Of the eight patient tumors injected, six patients were found to have identifiable sentinel nodes on scintigraphy. In two patients, no identifiable drainage of the radiotracer was found. In this small study, an identifiable sentinel node could be found in $75 \%$ of tumors. The authors suggest that this may be helpful in patients in which biopsy of the sentinel node could clarify the extent of lymphatic involvement, which could have diagnostic and therapeutic implications [31].

Ming and colleagues evaluated the utility in performing frozen section analysis of enlarged lymph nodes during $\mathrm{RN}$ for RCC [34]. They performed frozen section analysis on lymph nodes $>1 \mathrm{~cm}$, before undertaking an extended lymphadenectomy. Of 702 consecutive patients, 114 had evidence of enlarged lymph nodes or palpably enlarged nodes and underwent frozen section analysis. On final pathology, they found that 78 patients $(68.4 \%)$ with enlarged lymph nodes did not harbor cancer while 36 (31.6\%) did have nodal metastases. Of these 36 patients with nodal disease on final pathology, 32 had positive findings on frozen section, resulting in positive predictive value of $100 \%$ and negative predictive value of $95 \%$. The study concludes that it would be reasonable to avoid LND in patients with clinically localized RCC in whom frozen section analysis of enlarged lymph nodes reveals no evidence of malignant disease. However, this does not indicate any therapeutic advantage to the procedure in patients with lymph node disease.

\section{Outcomes of LND}

Patients with clinically localized RCC have not been shown to benefit from routine LND [1]. The EORTC 30881 trial confirmed that after appropriate clinical staging, in patients with clinical N0M0 disease, the incidence of unsuspected lymph-node metastases was only $4.0 \%$. When compared with nephrectomy alone, they showed no advantage to performing LND on patients with clinically localized disease with regards to overall survival, local regional progression, or distant progression. Of the 346 patients in whom LND was performed, 51 had palpably enlarged lymph nodes at the time of surgery. Of these, only 10 patients $(20 \%)$ had lymph node metastases. Remarkably, of the 311 remaining patients without palpable nodes, only 4 patients (1\%) were shown to have metastatic disease to their lymph nodes $(P<.001)$. The potential benefits of staging for these patients are also minimal. These statements are especially true for more lowrisk disease (T1-2, N0, M0).

The more difficult patient population to address is those with clinically localized, high-risk disease (T3-4, N0, M0). The root of this difficulty stems from the fact that there is substantial risk of hematogenous dissemination of disease, and relatively low risk of node-only involvement. The therapeutic benefit of LND in these patients is questionable at best. As previously discussed, Blute and colleagues identified 5 risk factors (including clinical stage T3-4) for lymph node metastases. The presence of at least 2 risk factors was associated with a 15-fold higher incidence of regional lymph node involvement. Although difficult to implement, this is reasonable approach for these patients in whom occult disease may be cured.

Patients who present with clinical nodal disease should have LND performed. It is relatively infrequent for patients to present with isolated positive nodes, without distant metastases, but is estimated to occur in $3 \%$ to $10 \%$ of cases $[1,17-19]$. It is essential, therefore, to accurately rule out distant metastatic disease if one suspects lymph node involvement only. Survival of these patients improves when LND is performed compared to nephrectomy alone [32]. Further, the overall survival of these patients who undergo LND with radical nephrectomy is far superior to patients who present with distant metastases, and in fact more closely approximates the survival of patients with T3, N0, M0 disease $[35,36]$. Giuliani and colleagues showed that 5-year survival in patients with lymph node only disease was $47.9 \%$, compared with $7 \%$ for patients with distant metastases. An extended lymph node dissection, as described previously, is recommended in these patients.

Patients with metastatic disease may benefit only slightly, if at all, from LND at the time of nephrectomy. Nodal metastases are poorly responsive to immunotherapy, so removal of grossly positive nodes is reasonable. Although extended lymphadenectomy may theoretically be beneficial, there is no evidence to support this, and lymphadenectomy must be balanced with the patient's comorbidities and performance status. A useful, evidence-based algorithm was offered by Godoy and colleagues [16] that can be utilized in the decision to perform LND at the time of radical nephrectomy. With 
the advent of new tyrosine kinase inhibitors and cytokines, the value of LND in patients with metastatic disease may increase in the near future, either as adjuvant or neoadjuvant therapy.

\section{Current State of Targeted Therapies}

Previously, systemic treatment of patients with metastatic RCC was limited to cytokine therapy with interleukin(IL-) 2 or interferon- (IFN-) $\alpha$, because of mRCC's general resistance to chemotherapy. High-dose IL-2 remains the only treatment to produce durable remissions, and it should be considered in healthy, appropriately chosen candidates as adjuvant therapy. New targeted therapies have been developed through genetic studies of familial von Hippel Lindau disease. Specifically, molecular cell signaling pathways involving the vascular endothelial growth factor (VEGF) and the mammalian target of rapamycin (mTOR) pathways have been targeted with tyrosine kinase inhibitors (TKIs) and mTOR inhibitors, respectively.

Node-only metastatic RCC is rare, and no specific data of these patients treated with targeted therapies currently exists. Instead, we suggest treating these patients similar to those having a solitary metastasis. Based on current data, sunitinib (TKI) is justified as a first-line standard of care for patients with clear cell RCC $[37,38]$. Another first-line therapy for clear cell RCC is combination therapy with bevacizumab plus interferon- $\alpha$ based on recent phase III data. Patients with nonclear cell metastatic RCC should be enrolled in clinical trials; however, they have been treated with TKIs, mTOR inhibitors, and even chemotherapy with gemcitabine and cisplatin for metastatic sarcomatoid RCC.

Currently, randomized controlled studies have been initiated to determine the current role and optimal timing of removal of the primary tumor in patients with primary metastatic disease, since historically cytoreductive nephrectomy followed by cytokine treatment had better overall survival than cytokine therapy alone. Therefore, participation in a clinical trial is currently considered as the best management of metastatic RCC patients presenting with a resectable primary and synchronous metastases. In patients with more advanced disease, anecdotal evidence has suggested that neoadjuvant targeted therapies disease can improve feasibility of cytoreductive nephrectomy, reduce nodal involvement, and facilitate lymphadenectomy by improving the planes of dissection [39]. We look forward to the further development of a targeted therapy regimen for patients with metastatic disease.

\section{Conclusion}

Lymphadenectomy at the time of radical nephrectomy is rarely performed and is not supported in the majority of patients with renal tumors, especially with the downward stage migration of disease. It does not appear to confer a survival advantage in patients who have clinically localized disease. However, LND at the time of nephrectomy is not associated with significantly increased morbidity [1] and is warranted if there is sufficient clinical suspicion on staging
CT, or if bulky lymphadenopathy is found at the time of surgery. Although lymphadenectomy undoubtedly improves the accuracy of staging and provides better prognostic information, there is little impact on progression-free or overall survival in patients with clinically localized disease. Risk factors may increase the likelihood of lymph node metastases, and may be a way to better determine patients at risk for nodal involvement. Future studies with novel targeted therapies may increase the indications for LND further.

\section{References}

[1] J. H. M. Blom, H. van Poppel, J. M. Maréchal et al., "Radical nephrectomy with and without lymph-node dissection: final results of European Organization for Research and Treatment of Cancer (EORTC) randomized phase 3 trial 30881," European Urology, vol. 55, no. 1, pp. 28-34, 2009.

[2] U. Capitanio, C. Jeldres, J. J. Patard et al., "Stage-specific effect of nodal metastases on survival in patients with nonmetastatic renal cell carcinoma," British Journal of Urology International, vol. 103, no. 1, pp. 33-37, 2009.

[3] B. C. Leibovich and M. L. Blute, "Lymph node dissection in the management of renal cell carcinoma," Urologic Clinics of North America, vol. 35, no. 4, pp. 673-678, 2008.

[4] A. Antonelli, A. Cozzoli, D. Zani et al., "The follow-up management of non-metastatic renal cell carcinoma: definition of a surveillance protocol," British Journal of Urology International, vol. 99, no. 2, pp. 296-300, 2007.

[5] T. Klatte, H. Wunderlich, J. J. Patard et al., "Clinicopathological features and prognosis of synchronous bilateral renal cell carcinoma: an international multicentre experience," British Journal of Urology International, vol. 100, no. 1, pp. 21-25, 2007.

[6] G. Verhoest, D. Veillard, F. Guillé et al., "Relationship between age at diagnosis and clinicopathologic features of renal cell carcinoma," European Urology, vol. 51, no. 5, pp. 1298-1305, 2007.

[7] C. Terrone, C. Cracco, F. Porpiglia et al., "Reassessing the current TNM lymph node staging for renal cell carcinoma," European Urology, vol. 49, no. 2, pp. 324-331, 2006.

[8] J. J. Patard, "Prognostic and biological significance of lymph node spreading in renal cell carcinoma," European Urology, vol. 49, no. 2, pp. 220-222, 2006.

[9] V. Ficarra, G. Novara, M. Iafrate et al., "Proposal for reclassification of the TNM staging system in patients with locally advanced (pT3-4) renal cell carcinoma according to the cancer-related outcome," European Urology, vol. 51, no. 3, pp. 722-731, 2007.

[10] V. Margulis, P. Tamboli, K. M. Jacobsohn, D. A. Swanson, and C. G. Wood, "Oncological efficacy and safety of nephronsparing surgery for selected patients with locally advanced renal cell carcinoma," British Journal of Urology International, vol. 100, no. 6, pp. 1235-1239, 2007.

[11] S. E. Delacroix and C. G. Wood, "The role of lymphadenectomy in renal cell carcinoma," Current Opinion in Urology, vol. 19, no. 5, pp. 465-472, 2009.

[12] U. E. Studer and F. D. Birkhäuser, "Lymphadenectomy combined with radical nephrectomy: to do or not to do?" European Urology, vol. 55, no. 1, pp. 35-37, 2009.

[13] S. H. Landis, T. Murray, S. Bolden, and P. A. Wingo, "Cancer statistics, 1999," CA-A Cancer Journal for Clinicians, vol. 49, no. 1, pp. 8-31, 1999. 
[14] A. J. Pantuck, A. Zisman, and A. S. Belldegrun, "The changing natural history of renal cell carcinoma," Journal of Urology, vol. 166, no. 5, pp. 1611-1623, 2001.

[15] W. H. Chow, S. S. Devesa, J. L. Warren, and J. F. Fraumeni Jr., "Rising incidence of renal cell cancer in the United States," Journal of the American Medical Association, vol. 281, no. 17, pp. 1628-1631, 1999.

[16] G. Godoy, R. L. O’Malley, and S. S. Taneja, "Lymph node dissection during the surgical treatment of renal cancer in the modern era," International Braz Journal Urology, vol. 34, no. 2, pp. 132-142, 2008.

[17] S. E. Canfield, A. M. Kamat, R. F. Sánchez-Ortiz, M. Detry, D. A. Swanson, and C. G. Wood, "Renal cell carcinoma with nodal metastases in the absence of distant metastatic disease (clinical stage TxN1-2M0): the impact of aggressive surgical resection on patient outcome," Journal of Urology, vol. 175, no. 3, pp. 864-869, 2006.

[18] A. Minervini, L. Lilas, M. Traversi et al., "Regional lymph node dissection in the treatment of renal cell carcinoma: is it useful in patients with no suspected adenopathy before or during surgery?" British Journal of Urology International, vol. 88, no. 3, pp. 169-172, 2001.

[19] J. R. Vasselli, J. C. Yang, W. M. Linehan, D. E. White, S. A. Rosenberg, and M. M. Walther, "Lack of retroperitoneal lymphadenopathy predicts survival of patients with metastatic renal cell carcinoma," Journal of Urology, vol. 166, no. 1, pp. 68-72, 2001.

[20] I. Frank, M. L. Blute, J. C. Cheville, C. M. Lohse, A. L. Weaver, and H. Zincke, "An outcome prediction model for patients with clear cell renal cell carcinoma treated with radical nephrectomy based on tumor stage, size, grade and necrosis: the SSIGN score," Journal of Urology, vol. 168, no. 6, pp. 23952400, 2002.

[21] B. C. Leibovich, M. L. Blute, J. C. Cheville et al., "Prediction of progression after radical nephrectomy for patients with clear cell renal cell carcinoma: a stratification tool for prospective clinical trials," Cancer, vol. 97, no. 7, pp. 1663-1671, 2003.

[22] B. C. Leibovich, J. C. Cheville, C. M. Lohse et al., "A scoring algorithm to predict survival for patients with metastatic clear cell renal cell carcinoma: a stratification tool for prospective clinical trials," Journal of Urology, vol. 174, no. 5, pp. 17591763, 2005.

[23] A. J. Pantuck, A. Zisman, F. Dorey et al., "Renal cell carcinoma with retroperitoneal lymph nodes: role of lymph node dissection," Journal of Urology, vol. 169, no. 6, pp. 20762083, 2003.

[24] P. L. Crispen, R. H. Breau, C. Allmer et al., "Lymph node dissection at the time of radical nephrectomy for high-risk clear cell renal cell carcinoma: indications and recommendations for surgical templates," European Urology, vol. 59, no. 1, pp. 18-23, 2011.

[25] G. Carmignani, E. Belgrano, P. Puppo et al., "Lymphadenectomy in renal cancer," in Cancer of the Prostate and Kidney, M. Pavone-Macaluso and P. H. Smith, Eds., pp. 645-650, Plenum Press, New York, NY, USA, 1983.

[26] J. P. Siminovitch, J. E. Montie, and R. A. Straffon, "Lymphadenectomy in renal adenocarcinoma," Journal of Urology, vol. 127, no. 6, pp. 1090-1091, 1982.

[27] M. L. Blute, B. C. Leibovich, J. C. Cheville, C. M. Lohse, and H. Zincke, "A protocol for performing extended lymph node dissection using primary tumor pathological features for patients treated with radical nephrectomy for clear cell renal cell carcinoma," Journal of Urology, vol. 172, no. 2, pp. 465469, 2004.
[28] G. C. Hutterer, J. J. Patard, P. Perrotte et al., "Patients with renal cell carcinoma nodal metastases can be accurately identified: external validation of a new nomogram," International Journal of Cancer, vol. 121, no. 11, pp. 2556-2561, 2007.

[29] U. E. Studer, S. Scherz, J. Scheidegger et al., "Enlargement of regional lymph nodes in renal cell carcinoma is often not due to metastases," Journal of Urology, vol. 144, no. 2 I, pp. 243$245,1990$.

[30] R. H. Thompson, G. V. Raj, B. C. Leibovich et al., "Preoperative nomogram to predict positive lymph nodes during nephrectomy for renal cell carcinoma paper," in Proceedings of the American Urological Association Annual Meeting, Orlando, Fla, USA, May 2008, abstract no. 603.

[31] A. Bex, L. Vermeeren, G. de Windt, W. Prevoo, S. Horenblas, and R. A. V. Olmos, "Feasibility of sentinel node detection in renal cell carcinoma: a pilot study," European Journal of Nuclear Medicine and Molecular Imaging, pp. 1117-1123, 2010.

[32] S.J. Freedland and J.B. Dekernion, "Role of lymphadenectomy for patients undergoing radical nephrectomy for renal cell carcinoma," Reviews Urology, vol. 5, pp. 191-195, 2003.

[33] C. K. Phillips and S. S. Taneja, "The role of lymphadenectomy in the surgical management of renal cell carcinoma," Urologic Oncology: Seminars and Original Investigations, vol. 22, no. 3, pp. 214-223, 2004.

[34] X. Ming, L. Ningshu, L. Hanzhong, H. Zhongming, and L. Tonghua, "Value for frozen section analysis of enlarged lymph nodes during radical nephrectomy for renal cell carcinoma," Urology, vol. 74, no. 2, pp. 364-368, 2009.

[35] L. Giuliani, C. Giberti, G. Martorana, and S. Rovida, "Radical extensive surgery for renal cell carcinoma: long-term results and prognostic factors," Journal of Urology, vol. 143, no. 3, pp. 468-473, 1990.

[36] P. C. Peters and G. L. Brown, "The role of lymphadenectomy in the management of renal cell carcinoma," Urologic Clinics of North America, vol. 7, no. 3, pp. 705-709, 1980.

[37] B. Shuch, S. B. Riggs, J. C. LaRochelle et al., "Neoadjuvant targeted therapy and advanced kidney cancer: observations and implications for a new treatment paradigm," British Journal of Urology International, vol. 102, no. 6, pp. 692-696, 2008.

[38] R. J. Motzer, T. E. Hutson, P. Tomczak et al., "Sunitinib versus interferon alfa in metastatic renal-cell carcinoma," New England Journal of Medicine, vol. 356, no. 2, pp. 115-124, 2007.

[39] R. J. Motzer, T. E. Hutson, P. Tomczak et al., "Overall survival and updated results for sunitinib compared with interferon alfa in patients with metastatic renal cell carcinoma," Journal of Clinical Oncology, vol. 27, no. 22, pp. 3584-3590, 2009. 


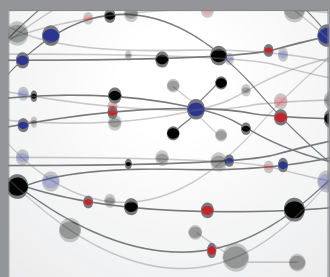

The Scientific World Journal
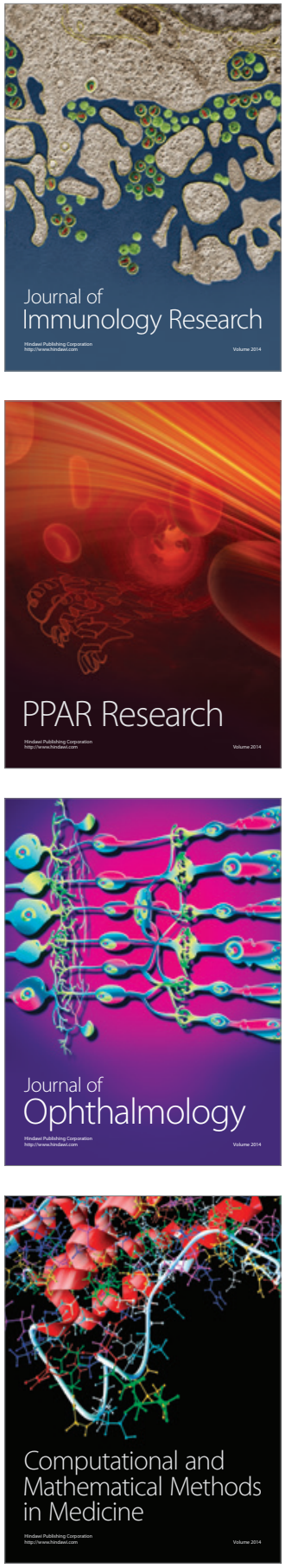

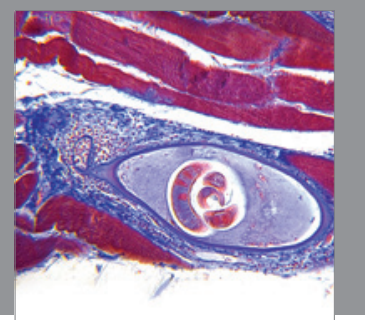

Gastroenterology

Research and Practice
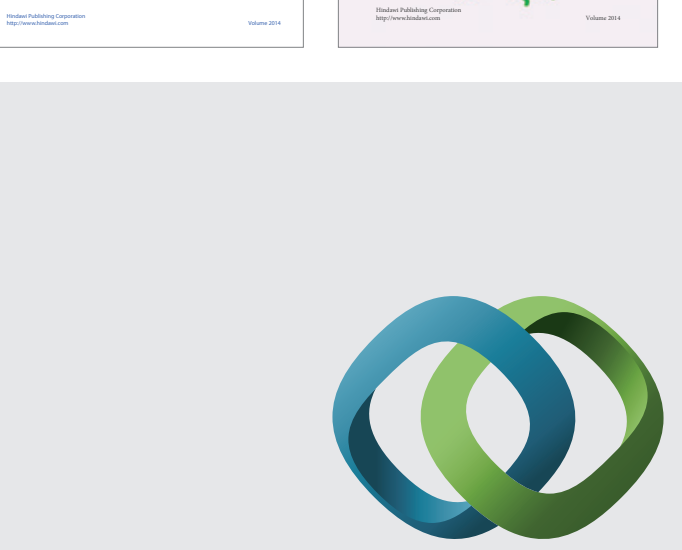

\section{Hindawi}

Submit your manuscripts at

http://www.hindawi.com
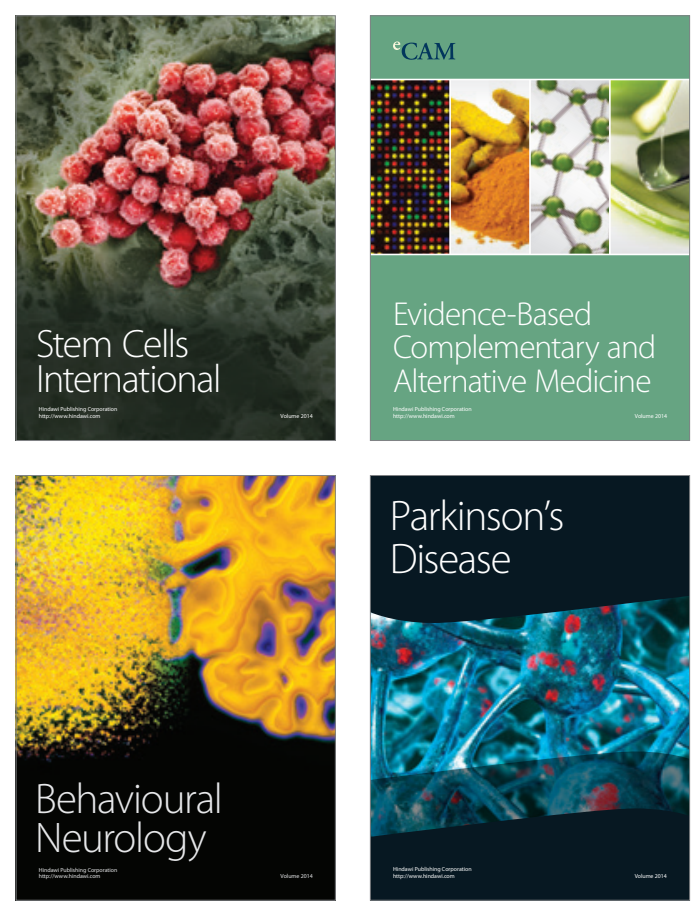

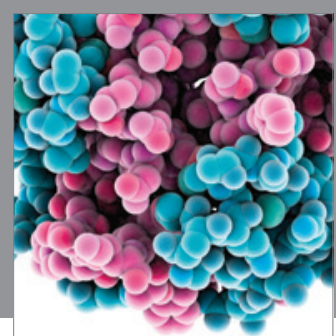

Journal of
Diabetes Research

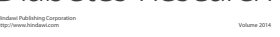

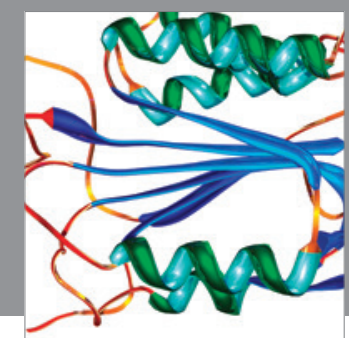

Disease Markers
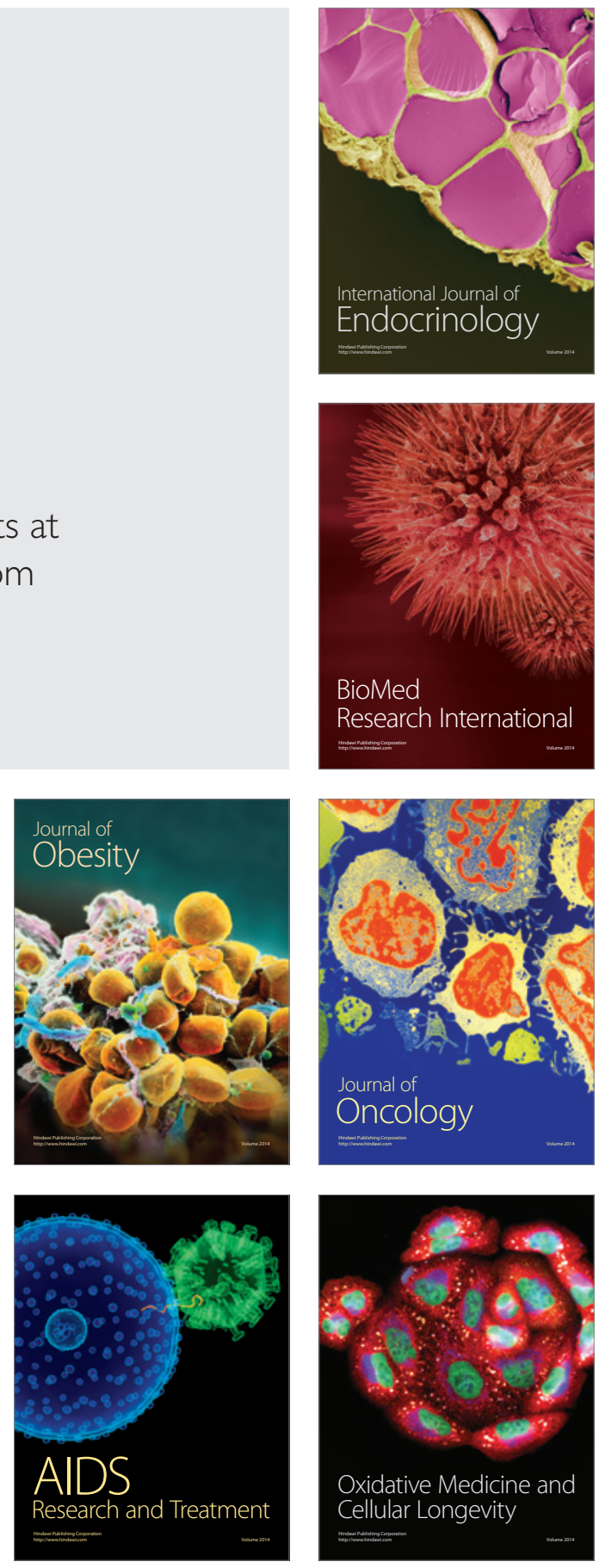